\title{
PROJECT OF ROTATING CARBON HIGH-POWER NEUTRON TARGET. RESEARCH OF GRAPHITE PROPERTIES FOR PRODUCTION OF HIGH INTENSITY NEUTRON SOURCE
}

\author{
M.S. Avilov, P.A. Bak, K.V. Gubin, N.Kh. Kot, P.V. Logatchev \\ Budker Institute of Nuclear Physics \\ 630090 Novosibirsk, Russia
}

\section{Abstract}

Results of MPG-class graphite properties research are presented. It is experimentally tested, that this kind of material can operate at high temperature conditions that are required for high power neutron target operation. The method of lifetime estimation is proposed. It is shown, that this material can stand under high temperature up to a few thousand hours.

\section{INTRODUCTION}

The proposed project of neutron source is based on the neutron target irradiated by the deuteron beam with energy $20 \mathrm{MeV}$, beam diameter $1 \mathrm{~cm}$, average power up to $100 \mathrm{~kW}$. The target represents the rotating disk with the operational area made of graphite. The target is cooled by its thermal radiation. Size, operational conditions, and lifetime are determined mainly by the maximum admissible temperature conditions of used material. The goal of the present paper is to define these conditions

\section{GRAPHITE PROPERTIES}

One can offer to use heat-resistant dense graphite MPG6 class as the target material. It is intended for the operation in vacuum or inert sphere at the temperature up to $2300-2500^{\circ} \mathrm{C}$. Its physical and mechanical properties are listed in Tab.1 (data are given by the manufacturing plant).

\section{EXPERIMENTS WITH GRAPHITE SAMPLES}

To test the graphite reliability at the target thermal conditions, series of experiments are done, aimed:

- to define the admissible number of thermocycles (fast heating up to operational temperature and fast cooling down to room temperature);

- to define the maximum admissible temperature gradient;

- $\quad$ to define the temperature jump influence;

- $\quad$ to define the lifetime in the temperature range 2200 $-2500^{\circ} \mathrm{C}$.
Table 1. Physics and mechanical properties of graphite MPG-6 class

\begin{tabular}{|l|l|}
\hline density, $\mathrm{g} / \mathrm{cm}^{3}$ & $1.76-1.88$ \\
\hline $\begin{array}{l}\text { ultimate strength at } 20^{\circ} \mathrm{C}, \mathrm{kN} / \mathrm{cm}^{2} \\
\text { compressive } \\
\text { cross-breaking } \\
\text { tensile }\end{array}$ & $10-12$ \\
\hline $\begin{array}{l}\left.\text { elastic modulus (dynamic, at } 20^{0} \mathrm{C}\right), \\
10^{3} \mathrm{kN} / \mathrm{cm}^{2}\end{array}$ & $5-7$ \\
\hline specific resistance at $20^{0} \mathrm{C}, \mathrm{Ohm} \cdot \mathrm{mm}^{2} / \mathrm{m}$ & $1.0-1.5$ \\
\hline $\begin{array}{l}\text { thermal conductivity ratio at } 1000^{0} \mathrm{C}, \\
\mathrm{kJ} /(\mathrm{m} \cdot \mathrm{h} \cdot \mathrm{K})\end{array}$ & $11-16$ \\
\hline $\begin{array}{l}\text { average temperature expansion ratio } \\
\text { in the range } 20-1500^{0} \mathrm{C}, 10^{-6} \cdot \mathrm{K}^{-1}\end{array}$ & $181-189$ \\
\hline radius of dominated pores, $\mu \mathrm{m}$ & $8.0-8.8$ \\
\hline total contents of admixtures & $3-8$ \\
\hline
\end{tabular}

To carry out these tests, a special testbench was made (see Fig.1). Graphite samples with the cross-section about $1.5 \times 1.5 \mathrm{~mm}$ and length $15-20 \mathrm{~mm}$ were heated in the vacuum volume by the pulsed current The pulse width was $96 \mu \mathrm{sec}$, the repetition rate was $50 \mathrm{~Hz}$. These conditions correspond to the operational conditions of the target, rotating with frequency $50 \mathrm{~Hz}$. Current and voltage oscillograms were registered, then radiated power, average temperature, and temperature jump were calculated. Control of the temperature distribution over the sample surface was also realized by the pirometer through the window in the vacuum volume. Upon the completion of the test the sample was then tested by the

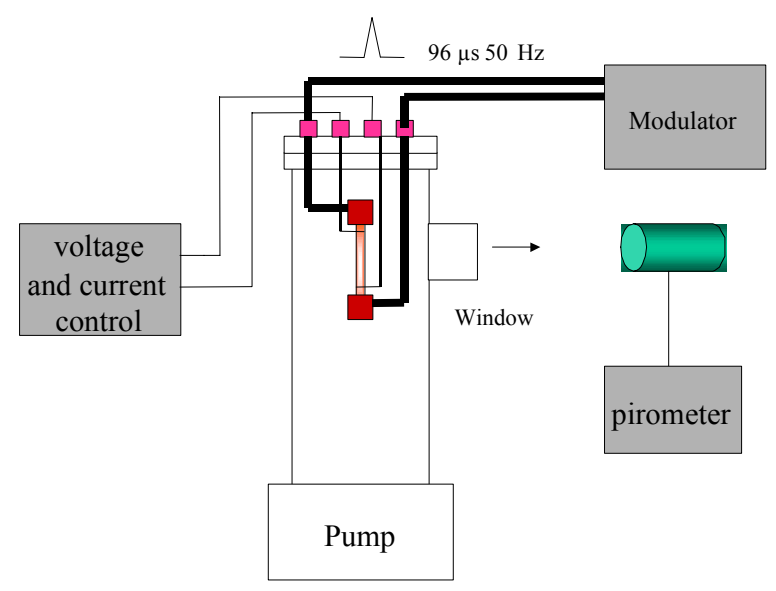

Figure 1. Installation layout 
electronic microscope.

Thermocycle test was carried out as follow: the sample was fed by the packet of heating current pulses of $30 \mathrm{sec}$ duration. It was heated up to the temperature $2000^{\circ} \mathrm{C}$. Then the next $30 \mathrm{sec}$ the sample was got cold down to the room temperature. As the result, the sample stood more than 500 cycles without destruction. Fig.2 show the protocol of the test. Note that for the first 200

cycles the sample resistance was risen up to $7 \%$ and then was stabilized on a new level, that point to stabilization of the sample material structure in a new level after its partial destruction, and success to stand the thermal stress of such a kind. This means the target doesn't require preliminary heating before the deuteron beam release.

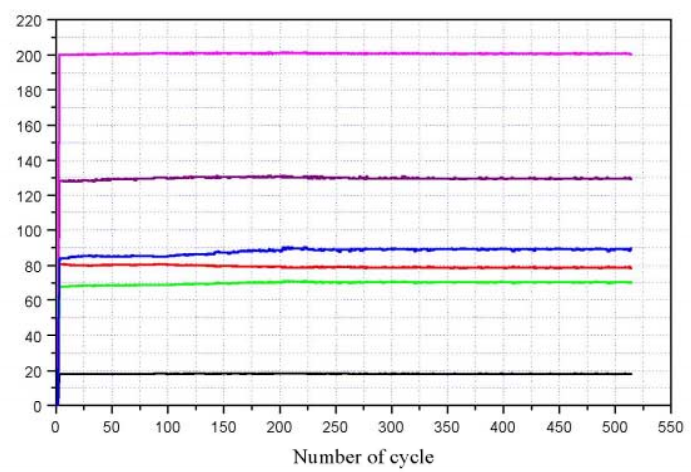

Figure 2. Protocol of graphite sample thermocycle test. Top to bottom: sample temperature in the end of heating $\mathrm{T}^{* 10}\left({ }^{0} \mathrm{C}\right)$, heating power $(\mathrm{W})$, sample resistance $(\mathrm{mOhm})$, current pulse amplitude (A), voltage pulse amplitude $(\mathrm{mV})$, temperature jump $\left({ }^{0} \mathrm{C}\right)$.

The measurements of the temperature distribution along the sample at quasi-stationary temperature $2000^{\circ} \mathrm{C}$ with the pirometer gave following results: maximum temperature was detected in the middle of $16 \mathrm{~mm}$ long sample. The temperature was $80^{\circ} \mathrm{C}$ lower at the edges of the sample. Thus, graphite stable stands the temperature gradient up to $100^{\circ} \mathrm{C} / \mathrm{cm}$.

To define the target lifetime the sample was heated by the current pulses and remained at constant average temperature in the range $2200-2500^{\circ} \mathrm{C}$. The change in time of the sample resistance indicated the change in graphite properties. The sample resistance was increased in time with the rising speed, and at $20-25 \%$ resistance growth the sample was destroyed. Time interval between the moment when the sample reached the operational temperature, and the moment when it was destroyed, was defined as the lifetime. Fig. 3 shows the protocol of the test for lifetime. The sample was destroyed after 1 hour while it stood at the average temperature around $2445^{\circ} \mathrm{C}$.

In order to define the temperature jump influence on the target lifetime the sample was heated by $50 \mathrm{~Hz}$ AC. Sample lifetime depended on the temperature only. On the other hand, the temperature jump at pulsed current heating
2-3 times exceeded the calculated one for normal operational conditions. That means that the main factor of target lifetime is its average operational temperature.

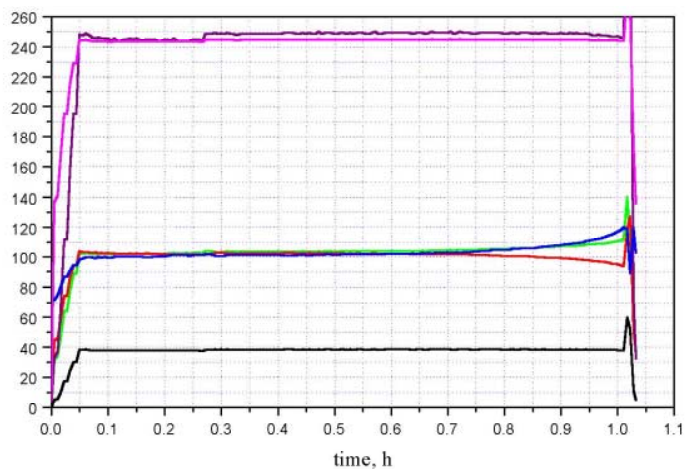

Figure 3. Protocol of graphite sample tested for the lifetime. Top to bottom: heating power (W), sample average temperature $\mathrm{T}^{*} 10 \quad\left({ }^{0} \mathrm{C}\right)$, sample resistance $(\mathrm{mOhm})$, voltage pulse amplitude $(\mathrm{V})$, current pulse amplitude I*10 (A), temperature jump $\left({ }^{0} \mathrm{C}\right)$.

\section{LIFETIME ESTIMATION - A MODEL}

One can use the following model for the interpretation of experiments: the sample material is porous, pores are long and narrow with typical transverse size $L$ and longitudinal $l$. Then the sample conductivity is $g \approx g_{0}(1-$ $S_{c} / S_{S}$ ), where $S_{c}=$ const $_{1} L l$ - the total area of the pores in the sample transverse cross-section, $S_{S}$ - the sample crosssection area. Pores are connected with the sample surface via their butt-ends of area $S_{c h} \approx$ const $_{2} L^{2}$. The graphite vapor is diffused from the pore surface through these buttends. The graphite vapor flux is $U \approx$ const $_{3} n V_{t} S_{c h}$, where $n$ - vapor concentration in pores, $V_{t}$ - mean thermal speed of the evaporated graphite. Since pores are long and narrow, one can assume that graphite vapor is saturated inside and then

$$
U \approx \frac{\text { const }_{4} L^{2} P_{C}}{\sqrt{T}}
$$

where $P_{c}$ - the saturated graphite vapor pressure at a given temperature $T$. On the other hand, graphite is evaporated from the pore surface changing its transverse size: $U \approx$ const $t_{5} S_{c} d L / d t$, where $L(t)=L_{0} \exp (A t)$ with

$$
\text { - } A=\operatorname{Const}_{C} / \sqrt{T}
$$

So, the measured experimental dependencies should be approximated by the formula:

$$
R(t)=R_{0} /(1-\text { const } \cdot \exp (A t))
$$

that seems to happen (see Fig.4).

The dependence of the graphite saturated vapor pressure $P_{C}=$ const $\cdot \exp (-B / T)$, where, according to 


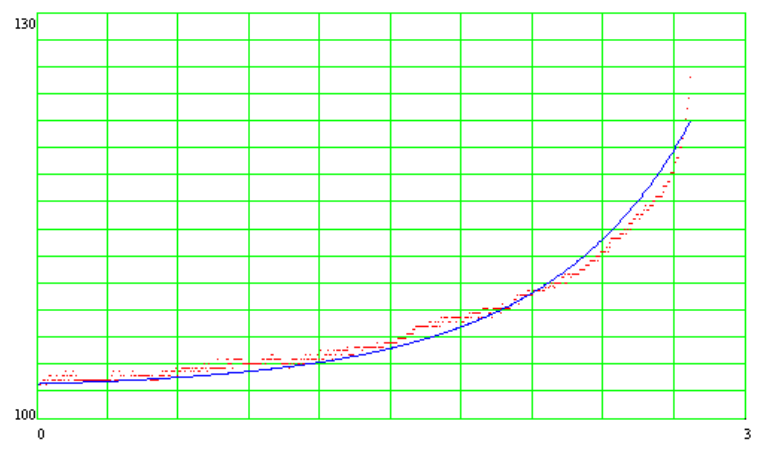

Figure 4. Dependence of sample resistance $(\mathrm{mOhm})$ on time (h) at temperature $2400^{\circ} \mathrm{C}$. Dots - measured values, solid line - approximation by formula (2.3)

various data $[1,2] B=9.2 \div 9.45 \cdot 10^{4}\left(T\right.$ measured in $\left.{ }^{0} \mathrm{~K}\right)$. In that case the sample lifetime depends on the temperature as follow:

$$
\tau=\text { Const } \sqrt{T} \exp (B / T)
$$

Fig. 5 shows the experimental data for samples lifetime dependence on temperature. The lifetime is determined after sample resistance $20 \%$ growth - at that time its strength (in the present model it depends on time the same way as the conductivity) is decreased for the same $20 \%$.

The experimental data are in good agreement with the curve gave by the formula (4) with Const $=3 \cdot 10^{-17}$. The lifetime defined in such a way at $2100^{\circ} \mathrm{C}$ appeared to be $300-400$ hours, about 2000 hours at $2000^{\circ} \mathrm{C}$, and about 10000 hours at the operational temperature $1800^{\circ} \mathrm{C}$.

\section{CONCLUSION}

From what has been said, it might be assumed, that graphite proves to be the appropriate material for the

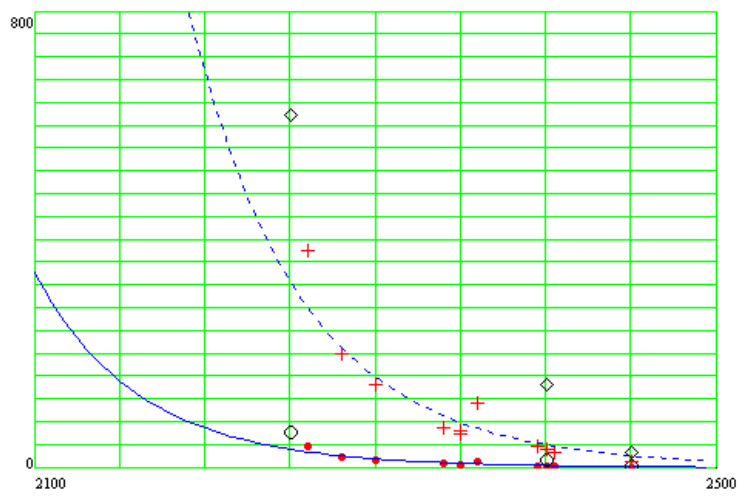

Figure 5. Dependence of samples lifetime (h) on temperature $\left({ }^{\circ} \mathrm{C}\right)$. $\bullet$ and $\mathrm{O}-$ measured values, + and $\diamond$ - same values multiplied by factor 10 , solid line approximation by formula (2.4), dashed line - same values multiplied by factor 10 .

neutron target irradiated by the deuteron beam of high power. It can stand under very high $\left(1800^{\circ}\right)$ temperature over a long period of time (a few thousand hours) Notice that the use of graphite as the basic target material essentially simplify the target and make it inexpensive, reliable and safe.

\section{REFERENCES}

[1] "Physics Values". Hand-book under reduction of I.S Grigoriev and E.Z. Melikhov. M. Energoatomizdat, 1991 (in Russian).

[2] T.Munsat. "High Temperature Carbon Irradiation for the Sombrero ICF Reactor". Princeton, NJ, Sept. 1999. 